\title{
N63A: A Supernova Remnant in a Cloudy Medium
}

\author{
You-Hua Chu, Adeline Caulet, John Dickel, Sean Points, Rosa Williams \\ Astronomy Department, University of Illinois, Urbana, IL 61801, USA
}

Lorena Arias-Montaño, Margarita Rosado

UNAM, Apdo Postal 70-264,04510 México DF, México

Patricia Ambrocio-Cruz, Annie Laval

Observatoire de Marseille, F-13248 Marseille Cedex 04, France

Dominik J. Bomans

Astronomisches Institut, Ruhr-Univ. Bochum, 44780 Bochum, Germany

\begin{abstract}
Our recently obtained HST WFPC2 images of N63A reveal shocked cloudlets in the SNR interior. The study of N63A helps us understand the structure and evolution of SNRs in a cloudy medium.
\end{abstract}

\section{N63A - A Puzzling SNR in an OB Association}

N63A was one of the first three supernova remnants (SNRs) discovered in the LMC based on its strong, nonthermal radio emission. Its identification as a SNR was subsequently confirmed by its bright X-ray emission and high $[\mathrm{S} \mathrm{II}] / \mathrm{H} \alpha$ ratios. As shown by the X-ray contours in Fig. 1a, N63A is located in the northeastern quadrant of the $\mathrm{H}$ II region $\mathrm{N} 63$ associated with the OB association LH 83. The progenitor of the supernova was likely a member of LH 83 (van den Bergh \& Dufour 1980). The currently most luminous star is an O7 V(ff)) star with a mass of $\sim 40 \mathrm{M}_{\odot}$ (Oey 1996). Therefore, the progenitor of N63A's supernova was probably more massive than $40 \mathrm{M}_{\odot}$ and its main-sequence spectral type earlier than 07 .

The spatial extent of N63A's X-ray emission is similar to that of its radio emission, $\sim 70^{\prime \prime}$, and defines the size of the SNR. Within the SNR's boundary, ground-based optical images show three bright nebular knots. Spectroscopic observations show that the two eastern knots have high $[\mathrm{S} \mathrm{II}] / \mathrm{H} \alpha$ ratios, indicating shock excitation, and that the western knot has a spectrum consistent with photoionization (Levenson et al. 1995). Besides these bright knots, no other optical features can be recognized as belonging to the SNR. The two [S II]-bright knots together extend over $\sim 23^{\prime \prime}$, much smaller than the SNR's size. The absence of optical emission from the rest of the remnant cannot be caused by foreground obscuration, as $\mathrm{A}_{\mathrm{v}}$ is generally $<0.5 \mathrm{mag}$ for stars in LH 83 (Oey 1996). Why is $N 63 A$ 's optical emission region so much smaller than the SNR's extent? 

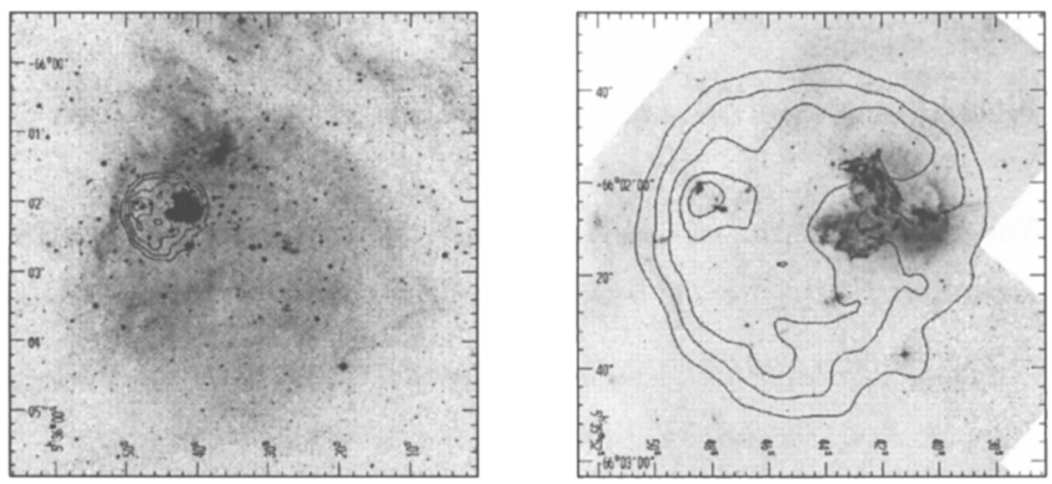

Figure 1. (a) $\mathrm{H} \alpha$ image of $\mathrm{N} 63$ overlaid by X-ray contours. (b) HST WFPC2 $\mathrm{H} \alpha$ image of N63A overlaid by X-ray contours.

\section{HST Images Reveal Shocked Clouds in N63}

The Hubble Space Telescope (HST) WFPC2 images of N63A in the H $\alpha$ and [SII] lines have revealed shocked clouds with sizes ranging from 5 pc down to 0.1 pc (Fig. 1b). N63A is clearly in a cloudy medium. The three bright nebular knots identified in ground-based images are well resolved in the WFPC2 images. The two eastern knots show distinct filamentary structures indicative of a compression by SNR shocks, while the western knot shows only diffuse ionized gas and dust clouds typical for H II regions. The two eastern knots might belong to the same cloud; their combined size is $\sim 5 \mathrm{pc}$, comparable to the Orion Nebula.

Within the SNR boundary, numerous [S II]-bright cloudlets are detected. The high $[\mathrm{S} \mathrm{II}] / \mathrm{H} \alpha$ ratios indicate that these cloudlets have been shocked and are located in the SNR interior. Some cloudlets show a diffuse morphology with radial density gradients, suggestive of isotropic evaporation and implying that the magnetic field is either very weak or tangled. Some cloudlets contain multiple cores, each $\sim 0.07 \mathrm{pc}$ across. The rms densities of the cloudlets range from 150 to $700 \mathrm{H} \mathrm{cm}^{-3}$, and the masses range from 0.01 to $\sim 1 \mathrm{M}_{\odot}$.

ROSAT and ASCA observations of N63A were used to derive a SNR interior $\mathrm{T}_{e}$ of $\sim 6 \times 10^{6} \mathrm{~K}, \mathrm{~N}_{e}$ of $\sim 5 \mathrm{~cm}^{-3}$, and a mass of $\sim 160 \mathrm{M}_{\odot}$. In such a hot medium, the evaporation timescale for the embedded cloudlets is a few $\times 10^{3}$ $\mathrm{yr}$, comparable to the dynamic age of N63A. It is conceivable that a significant amount of cloud mass has already been injected into the hot SNR interior through cloud evaporation.

\section{References}

Levenson, N. A., Kirshner, R. P., Elair, W. P., \& Winkler, P. F. 1995, PASP, 110,739

Lucke, P. B., \& Hodge, P. W. 1970, AJ, 75, 131

Oey, M. S. 1996, ApJ, 465, 231

van den Bergh, S., \& Dufour, R. J. 1980, PASP, 92, 32 